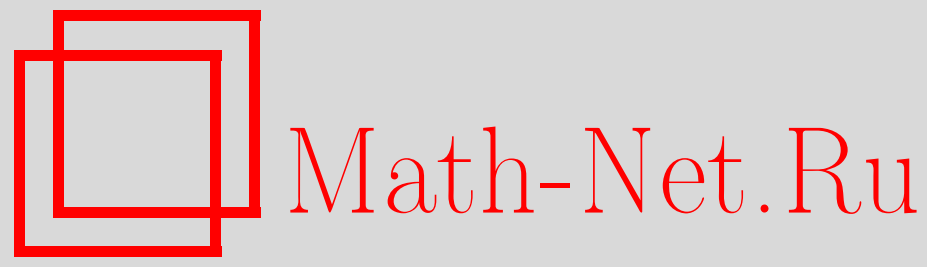

С. А. Назаров, О существенном спектре краевых задач для систем дифференциальных уравнений в ограниченной области с пиком, Функи. анализ и его прил., 2009, том 43, выпуск 1, 55-67

DOI: https://doi.org/10.4213/faa2934

Использование Общероссийского математического портала Math$\mathrm{Net.Ru}$ подразумевает, что вы прочитали и согласны с пользовательским соглашением

http://www . mathnet.ru/rus/agreement

Параметры загрузки:

IP : 18.234 .156 .22

26 апреля 2023 г., 13:52:35

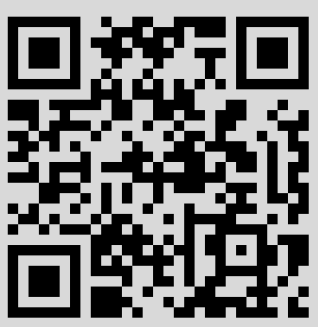


Функционалъный анализ и его приложения

2009, т. 43, вып. 1, с. 55-67

УДК 517.946

\title{
О существенном спектре краевых задач для систем дифференциальных уравнений в ограниченной области с пиком*
}

\author{
(C) 2009. C. A. НАзАров
}

\begin{abstract}
Найдены простые алгебраические условия появления существенного спектра оператора задачи Неймана для формально самосопряженной эллиптической системы дифференциальных уравнений в области с пикообразным заострением. В скалярном случае спектр оказывается дискретным.
\end{abstract}

\section{§1. Постановка задачи}

Известны (см., например, [1]) ограниченные области $\Omega$, для которых нет компактного вложения пространства Соболева $H^{1}(\Omega)$ в пространство Лебега $L_{2}(\Omega)$. Как следствие [2, теорема 10.1.5], спектр задачи Неймана для оператора Лапласа в таких областях $\Omega$ не может быть дискретным. У этих областей довольно экзотическое строение, и они, разумеется, не являются липшицевыми: в областях с липшицевыми границами вложение $H^{1}(\Omega) \subset L_{2}(\Omega)$ компактно и спектр задачи Неймана дискретен. Вместе с тем потеря липшицевости границей $\partial \Omega$ не обязательно влечет за собой возникновение существенного спектра. Так, если у области $\Omega \subset \mathbb{R}^{n}$ граница $\partial \Omega$ липшицева всюду, кроме точки $\mathscr{O}-$ начала декартовой системы координат $x=(y, z) \in \mathbb{R}^{n-1} \times \mathbb{R}$, а внутри шара $\mathbb{B}_{R}^{n}=\{x:|x|<R\}$ с радиусом $R>0$ эта область определена соотношениями (см. рисунок ниже)

$$
z>0, \quad \eta:=z^{-1-\gamma} y \in \omega \subset \mathbb{R}^{n-1},
$$

то при $\gamma>0$ поверхность $\partial \Omega$ не удовлетворяет условию Липшица, однако спектр задачи Неймана для оператора Лапласа дискретен (см. далее комментарий к теореме 1). В формуле (1) $\omega$ - область с компактным замыканием $\bar{\omega}$ и липшицевой границей $\partial \omega$, a $\gamma>0-$ показатель заострения пика. Сечение $\{x: \eta \in \omega, z=t\}$ пика (1) обозначаем через $\omega(t)$.

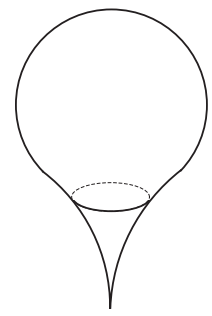

*Работа выполнена при финансовой поддержке Netherlands Organization for Scientific Research и Российского фонда фундаментальных исследований, совместный проект 047.017.020. 
В статье установлено, что в случае, когда граница имеет особенность простого геометрического строения (1), скалярные краевые задачи имеют дискретный спектр, но у краевых задач для систем дифференциальных уравнений существенный спектр не обязательно пустой - найден признак его образования для систем, обладающих полиномиальным свойством ([3], [4]). Этот признак превращается в критерий для достаточно острых $(\gamma \geqslant 1)$ пиков.

В области $\Omega$ рассмотрим краевую задачу Неймана ${ }^{1)}$ для системы уравнений второго порядка в дивергентной форме

$$
\begin{aligned}
& \mathscr{L}\left(x, \nabla_{x}\right) u(x):={\overline{\mathscr{D}\left(-\nabla_{x}\right)}}^{\top} A(x) \mathscr{D}\left(\nabla_{x}\right) u(x)=\lambda B(x) u(x), \quad x \in \Omega, \\
& \mathscr{N}\left(x, \nabla_{x}\right) u(x):=\overline{\mathscr{D}}(\nu(x))^{\top} A(x) \mathscr{D}\left(\nabla_{x}\right) u(x)=0, \quad x \in \partial \Omega \backslash \mathscr{O} .
\end{aligned}
$$

Здесь $A$ и $B$ - эрмитовы матрицы-функции размеров $N \times N$ и $k \times k$ соответственно, измеримые, а также ограниченные и равномерно положительно определенные почти всюду на множестве $\Omega$. Кроме того, $\mathscr{D}\left(\nabla_{x}\right)$ есть $(N \times k)$-матрица дифференциальных операторов первого порядка с постоянными коэффициентами, причем $N \geqslant k$. Символ ${ }^{\top}$ означает транспонирование, а черта - комплексное сопряжение, т. е. ${\overline{\mathscr{D}}\left(\nabla_{x}\right)}^{\top}$ - формально сопряженный оператор для $\mathscr{D}\left(\nabla_{x}\right)$ и $\mathscr{L}\left(x, \nabla_{x}\right)$ - формально самосопряженный матричный дифференциальный оператор. В левом сомножителе матричного оператора $\mathscr{N}\left(x, \nabla_{x}\right)$ градиент $\nabla_{x}$ заменен единичным вектором $\nu$ внешней нормали к поверхности $\partial \Omega \backslash \mathscr{O}$. Мы называем $\mathscr{N}$ оператором краевых условий Неймана потому, что вариационная формулировка задачи (2), (3)

$$
a(u, v ; \Omega):=\left(A \mathscr{D}\left(\nabla_{x}\right) u, \mathscr{D}\left(\nabla_{x}\right) v\right)_{\Omega}=\lambda(B u, v)_{\Omega}, \quad v \in C_{c}^{\infty}(\bar{\Omega} \backslash \mathscr{O})^{k},
$$

не требует ограничений на значения пробной вектор-функции $v=\left(v_{1}, \ldots, v_{k}\right)^{\top}$ на поверхности $\partial \Omega \backslash \mathscr{O}$; здесь $(\cdot, \cdot)_{\Omega}$ - скалярное произведение в (комплексном) пространстве $L_{2}(\Omega)^{m}$ при $m=N$ или $m=k$ - размеры векторов не указываются в обозначениях норм и скалярных произведений, а $C_{c}^{\infty}(\bar{\Omega} \backslash \mathscr{O})^{k}-$ линейное пространство гладких вектор-функций с компактными носителями, отделенными от точки $\mathscr{O}$.

Предположим, что матрица $\mathscr{D}$ является алгебраически комплектной [5, гл. 5]: существует такое натуральное число $\rho_{\mathscr{D}}$, что для каждой строки $P(\xi)=\left(P_{1}(\xi)\right.$, $\left.\ldots, P_{k}(\xi)\right)$ однородных степени $\rho \geqslant \rho \mathscr{D}$ полиномов переменных $\xi=\left(\xi_{1}, \ldots, \xi_{n}\right)^{\top}$ найдется полиномиальная строка $Q(\xi)=\left(Q_{1}(\xi), \ldots, Q_{N}(\xi)\right)$, удовлетворяющая соотношению

$$
P(\xi)=Q(\xi) \mathscr{D}(\xi), \quad \xi \in \mathbb{R}^{n} .
$$

Согласно [3], [4], соотношение (5) обеспечивает полиномиальное свойство полуторалинейной формы $а$ из интегрального тождества (4), т. е. соответствующий квадратичный функционал вырождается только на конечномерном линеале $\mathscr{P}$ векторных полиномов,

$$
\mathscr{P}=\left\{p=\left(p_{1}, \ldots, p_{k}\right)^{\top}: \mathscr{D}\left(\nabla_{x}\right) p(x)=0, x \in \mathbb{R}^{n}\right\} .
$$

На основании формулы (5) нетрудно убедиться в том, что степени скалярных полиномов $p_{j}$, фигурирующих в определении $(6)$, не превосходят $\rho_{\mathscr{D}}-1$.

1) Разумеется, в отличие от вариационной постановки (4) классическая постановка задачи требует дополнительной гладкости данных. 


\section{§2. Признак и критерий наличия существенного спектра}

Введем новый спектральный параметр

$$
\mu=(1+\lambda)^{-1}
$$

и перепишем интегральное тождество (4) как абстрактное уравнение

$$
\mathscr{T} u=\mu u
$$

в гильбертовом пространстве $\mathscr{H} \subset L_{2}(\Omega)^{k}$, полученном пополнением линеала $C_{c}^{\infty}(\bar{\Omega} \backslash \mathscr{O})^{k}$ по естественной норме

$$
\|u ; \mathscr{H}\|=(a(u, u ; \Omega)+b(u, u ; \Omega))^{1 / 2},
$$

где $b(u, v ; \Omega):=(B u, v)_{\Omega}, u, v \in \mathscr{H}$. Оператор $\mathscr{T}$ задан формулой

$$
\langle\mathscr{T} u, v\rangle=b(u, v ; \Omega), \quad u, v \in \mathscr{H} .
$$

Здесь $\langle\cdot, \cdot\rangle$ - скалярное произведение, порожденное нормой (9). Понятно, что $\mathscr{T}$ - положительный, непрерывный и симметрический оператор, а значит, самосопряженный. Его норма равна единице, и число $\mu=1$ принадлежит точечному спектру, а соответствующее собственное подпространство совпадает с линеалом (6). По понятным причинам множество $\mathbb{C} \backslash \overline{\Upsilon_{0}^{1}}$ содержится в резольвентном множестве оператора $\mathscr{T} ;$ здесь $\Upsilon_{a}^{b}=\{\lambda \in \mathbb{C}: \operatorname{Im} \lambda=0, a<\operatorname{Re} \lambda \leqslant b\}$ полуинтервал на вещественной оси.

По замыканию интегральное тождество (4) распространяется на пробные функции $v \in \mathscr{H}$. Поэтому задачи (4) и (8) эквивалентны, а связь (7) спектральных параметров передает свойства спектра одной из них спектру другой. Далее мы в основном имеем дело с оператором $\mathscr{T}$.

Сформулируем центральный результат работы, доказательство которого проводится в следующих параграфах.

Теорема 1. 1) Oператор Я компактен при любом $\gamma>0$ (и, следовательно, его дискретный спектр лежит на вещественной положстельной полуоси) тогда и только тогда, когда линеал (6) состоит из векторных полиномов $p$, не зависящих от переменной z.

2) При наличии в линеале ЯР полинома

$$
p(y, z)=z^{J} p^{0}(y)+\cdots+z p^{J-1}(y)+p^{J}(y),
$$

у которого $J \geqslant 1$ и $p^{0} \neq 0$, вложение $\mathscr{H} \subset L_{2}(\Omega)^{k}$ не является компактным в случае $\gamma \geqslant J^{-1}$, m.е. на полуинтервале $\Upsilon_{0}^{1}$ оператор $\mathscr{T}$ имеет непустой существенный спектр. Если $\gamma>J^{-1}$, то собственное число $\mu=1$ этого оператора с (конечномерным) собственным подпространством (6) принадлежит непрерывному спектру оператора $\mathscr{T}$.

Замечание 2. Общие результаты теории эллиптических краевых задач в областях с кусочно гладкими границами (см., например, [6]) показывают, что оператор задачи (2), (3) является фредгольмовым в пространствах со специальными нормами, которые содержат весовые множители, экспоненциально малые вблизи вершины пика $\mathscr{O}$. «Энергетическое» пространство $\mathscr{H}$ содержится в этих весовых пространствах, а значит, ядро оператора $\mathscr{T}$ оказывается конечномерным и его существенный спектр совпадает с непрерывным. Впрочем, это наблюдение не играет роли в дальнейших рассмотрениях. 
Для задачи (4) первое утверждение теоремы означает, что ее собственные числа образуют бесконечно большую последовательность

$$
0=\lambda_{1}=\cdots=\lambda_{d}<\lambda_{d+1} \leqslant \ldots \leqslant \lambda_{q} \leqslant \cdots+\infty,
$$

в которой они указаны с учетом кратностей. В частности, $d=\operatorname{dim} \mathscr{P} \geqslant k$, так как заведомо $\mathbb{C}^{k} \subset \mathscr{P} \subset \mathscr{H}$.

Если $\mathscr{L}$ - скалярный дифференциальный оператор, то $\mathscr{D}(\xi)$ - столбец высоты $N \geqslant n$ и $\left|\nabla_{x} u(x)\right| \leqslant c\left|\mathscr{D}\left(\nabla_{x}\right) u(x)\right|$ для любой гладкой функции $u$, т. е. $\mathscr{P}=\mathbb{C}$ и скалярная задача Неймана (4) при любом показателе заострения $\gamma>0$ обладает дискретным спектром (12). В $\S 5$ приведены примеры квадратичных форм $a(u, v ; \Omega)$, для которых в линеале $\mathscr{P}$ имеются полиномы вида (11) при $J \geqslant 1$.

\section{§3. Существенный и непрерывный спектры}

Заметим, что линеал (6) инвариантен относительно сдвигов $x \mapsto x+\tau$ и растяжений $x \mapsto \sigma x$ декартовой системы координат (здесь $\tau \in \mathbb{R}^{n}$ и $\sigma \in \mathbb{R}_{+}$). Применяя эти преобразования с подходящими наборами параметров $\tau$ и $\sigma$, видим, что вместе с векторным полиномом $p$ из (11) все входящие в него однородные векторные полиномы и все его производные также принадлежат линеалу $\mathscr{P}$. Таким образом, предположение в п. 2) теоремы 1 означает, что в $\mathscr{P}$ есть столбец однородных полиномов степени $J$, который представим в виде

$$
P(y, z)=\sum_{j=0}^{J} \frac{J !}{j !} P^{j}(y) z^{J-j}=\sum_{j=0}^{J} P^{j}(y) \frac{\partial^{j} z^{J}}{\partial z^{j}}, \quad \operatorname{deg} P^{j}=j, \quad P^{0} \in \mathbb{C}^{k} \backslash\{0\} .
$$

Выполнив дифференцирование, нетрудно убедиться в том, что равенство $\mathscr{D}\left(\nabla_{y}, \partial_{z}\right) P(y, z)=0$ эквивалентно соотношениям

$$
\mathscr{D}\left(\nabla_{y}, 0\right) P^{J-j}(y)=-\mathscr{D}(0, \ldots, 0,1) P^{J-j-1}(y), \quad j=0, \ldots, J,
$$

где $P^{-1}=0$ и $\nabla_{y}=\left(\partial / \partial y_{1}, \ldots, \partial / \partial y_{n-1}\right)^{\top}$.

Пусть $\psi$ - нетривиальная бесконечно дифференцируемая функция переменной $z$ с носителем на сегменте $[1,2]$. Положим $\psi^{q}(z)=\psi\left(2^{q} z\right)$ и $\Psi^{q}(y, z)=$ $P\left(y, \partial_{z}\right) \psi^{q}(z)$, где $q=1,2, \ldots$ и $\partial_{z}=\partial / \partial z$. Ясно, что $\psi^{p} \psi^{q}=0$ при $p \neq q$. В силу соотношений (14) получаем

$$
\begin{aligned}
\mathscr{D}\left(\nabla_{x}\right) \Psi^{q}(x) & =\sum_{j=0}^{J} \mathscr{D}\left(\nabla_{y}, 0\right) P^{j}(y) \partial_{z}^{j} \psi^{q}(z)+\sum_{j=0}^{J} \mathscr{D}(0, \ldots, 0,1) P^{j}(y) \partial_{z}^{j+1} \psi^{q}(z) \\
& =\mathscr{D}(0, \ldots, 0,1) P^{J}(y) \partial_{z}^{J+1} \psi^{q}(z) .
\end{aligned}
$$

Итак, при помощи формул (13) находим, что при $|y| \leqslant c z^{1+\gamma}$ и $z \in\left(2^{-q}, 2^{1-q}\right)$ верны оценки

$$
\left|\Psi^{q}(x)\right| \geqslant c_{1}\left|\psi^{q}(z)\right|-c_{2} \sum_{j=1}^{J} 2^{q j} z^{(1+\gamma) j}, \quad\left|\mathscr{D}\left(\nabla_{x}\right) \Psi^{q}(x)\right| \leqslant c_{3} 2^{q(J+1)} z^{(1+\gamma) J},
$$

а при $z \notin\left(2^{-q}, 2^{1-q}\right)$ вектор-функция $\Psi^{q}$ равна нулю. Здесь и далее $c_{m}$ положительные постоянные, зависящие от полинома $P$ и функции $\psi$, но не зависящие от параметра $q$ и координаты $z$. Выполнив интегрирование по части 
множества, заданной формулами (1), обнаруживаем, что при большом натуральном $q$ справедливы неравенства

$$
\begin{gathered}
\left\|\Psi^{q} ; L_{2}(\Omega)\right\|^{2} \geqslant c_{4} 2^{-(n(1+\gamma)-\gamma) q} \\
\left\|\mathscr{D}\left(\nabla_{x}\right) \Psi^{q} ; L_{2}(\Omega)\right\|^{2} \leqslant c_{5} 2^{-(n(1+\gamma)-\gamma) q} 2^{2(J+1) q} 2^{-2(1+\gamma) J q} .
\end{gathered}
$$

Если $\gamma=J^{-1}$, то правые части формул (15) являются бесконечно малыми одного порядка при $q \rightarrow+\infty$, а значит, вектор-функции $\Phi^{q}=2^{(n(1+\gamma)-\gamma) q / 2} \Psi^{q}$ образуют ограниченную последовательность элементов пространства $\mathscr{H}$, слабо сходящуюся в $\mathscr{H}$ к нулю, но не сходящуюся сильно в пространстве $L_{2}(\Omega)^{k}$. Иными словами, вложение $\mathscr{H} \subset L_{2}(\Omega)^{k}$ не является компактным, а спектр оператора $\mathscr{T}$ на полуинтервале $\Upsilon_{0}^{1}-$ дискретным.

В силу формул (9), (10) и (15) справедливо соотношение

$$
\begin{aligned}
\left\|\mathscr{T} \Phi^{q}-\Phi^{q} ; \mathscr{H}\right\| & =\sup \left|\left\langle\mathscr{T} \Phi^{q}-\Phi^{q}, v\right\rangle\right|=\sup \left|\left(A \mathscr{D}\left(\nabla_{x}\right) \Phi^{q}, \mathscr{D}\left(\nabla_{x}\right) v\right)_{\Omega}\right| \\
& \leqslant c 2^{(n(1+\gamma)-\gamma) q / 2}\left\|\mathscr{D}\left(\nabla_{x}\right) \Psi^{q} ; L_{2}(\Omega)\right\| \leqslant c 2^{(1-\gamma J) q}
\end{aligned}
$$

где верхняя грань вычисляется по таким $v \in \mathscr{H}$, что $\|v ; \mathscr{H}\|=1$. Таким образом, при $\gamma>J^{-1}$ последовательность $\left\{\Phi^{q}\right\}$ сингулярна для самосопряженного оператора $\mathscr{T}$ в точке $\mu=1$, и по критерию Вейля (см., например, [2, §9.1]) эта точка принадлежит существенному спектру, а значит, и непрерывному, так как $\mu=1$ - конечнократное собственное число.

Второе утверждение теоремы 1 доказано.

Отметим, что

$$
\left|\partial_{z} \Psi^{q}(x)\right| \leqslant c_{6} 2^{q}, \quad\left\|\nabla_{x} \Psi^{q} ; L_{2}(\Omega)\right\|^{2} \geqslant c_{7} 2^{-(n(1+\gamma)-\gamma) q} 2^{2 q},
$$

т. е. в случае $J \geqslant 1$ вложения $\mathscr{H} \subset H^{1}(\Omega)^{k}$ нет ни при каком положительном показателе $\gamma$.

\section{§4. Весовое неравенство Корна}

Начнем с проверки вспомогательного алгебраического факта.

Лемма 3. Если в линеале ЯР нет полиномов, зависящих от переменной $z$, то при любъх столбцах $a^{1}, \ldots, a^{n} \in \mathbb{C}^{k}$ выполнено соотношение

$$
\left|\sum_{j=1}^{n-1} \mathscr{D}\left(e_{j}\right) a^{j}\right|^{2}+\left|\mathscr{D}\left(e_{n}\right) a^{n}\right|^{2} \leqslant c_{\mathscr{D}}\left|\sum_{p=1}^{n} \mathscr{D}\left(e_{p}\right) a^{p}\right|^{2},
$$

в котором $e_{j}$ - элемент стандартного базиса в евклидовом пространстве $\mathbb{R}^{n}$, а с⿻ - постоянная, зависящая от матрищъ $\mathscr{D}$, но не зависящая от столбцов $a^{p}$.

Доказательство. Рассмотрим в пространстве $\mathbb{C}^{N}$ следующие линейные оболочки столбцов $\mathscr{D}^{1}\left(e_{p}\right), \ldots, \mathscr{D}^{k}\left(e_{p}\right)$ матриц $\mathscr{D}\left(e_{p}\right)$ :

$$
\begin{aligned}
& \Pi_{y}=\operatorname{span}\left\{\mathscr{D}^{q}\left(e_{j}\right) \mid q=1, \ldots, k, j=1, \ldots, n-1\right\}, \\
& \Pi_{z}=\operatorname{span}\left\{\mathscr{D}^{q}\left(e_{n}\right) \mid q=1, \ldots, k\right\} .
\end{aligned}
$$

Поскольку пространство конечномерно, соотношение (16) обеспечено формулой $\Pi_{y} \cap \Pi_{z}=\{0\} \in \mathbb{C}^{N}$. Если предположить, что имеется нетривиальный столбец 
$a \in \Pi_{y} \cap \Pi_{z}$, то найдутся столбцы $a^{1}, \ldots, a^{n} \in \mathbb{C}^{k}$, удовлетворяющие соотношениям $a^{n} \neq 0$ и

$$
a=\mathscr{D}\left(e_{n}\right) a^{n}=-\sum_{j=1}^{n-1} \mathscr{D}\left(e_{j}\right) a^{j},
$$

а значит, справедливо равенство $\mathscr{D}\left(\nabla_{x}\right)\left(z a^{n}+y_{1} a^{1}+\cdots+y_{n-1} a^{n-1}\right)=0$, т. е. в линеале (6) есть нетривиальная линейная вектор-функция переменной $z$. Полученное противоречие заканчивает проверку неравенства (16).

Из следующего утверждения будет выведена компактность вложения $\mathscr{H} \subset$ $L_{2}(\Omega)^{k}$.

Теорема 4. Если в линеале ЯР нет полиномов, зависящих от переменной $z$, то справедливо весовое неравенство Корна

$$
\left\|r^{-1} v ; L_{2}(\Omega)\right\|^{2} \leqslant c\left(\left\|\mathscr{D}\left(\nabla_{x}\right) v ; L_{2}(\Omega)\right\|^{2}+\left\|v ; L_{2}(\Omega)\right\|^{2}\right),
$$

әде $r=|x|$, а $c$ - постоянная, зависящая от матрищь $\mathscr{D}$ и области $\Omega$ c пиком (1), но не зависящая от вектор-функции $v \in \mathscr{H}$.

Доказательство. Пусть $\Omega_{\delta}=\Omega \backslash \overline{\Xi_{\delta}}$ и $\Xi_{\delta}=\left\{x \in \Omega \cap \mathbb{B}_{R}^{n}: z<\delta\right\}$, где $\delta-$ малое положительное число. Область $\Omega_{\delta / 2}$ с обломанным кончиком пика имеет липшицеву границу, и, согласно [5], выполнено обобщенное неравенство Корна

$$
\left\|v ; H^{1}\left(\Omega_{\delta / 2}\right)\right\|^{2} \leqslant c_{\delta}\left(\left\|\mathscr{D}\left(\nabla_{x}\right) v ; L_{2}\left(\Omega_{\delta / 2}\right)\right\|^{2}+\left\|v ; L_{2}\left(\Omega_{\delta / 2}\right)\right\|^{2}\right) \leqslant c_{\delta}\|v ; \mathscr{H}\|^{2} .
$$

Умножим $v$ на гладкую срезающую функцию $\chi_{\delta}$, равную единице на множестве $\Xi_{\delta / 2}$ и нулю вне $\Xi_{\delta}$. В силу неравенства (18) верна оценка $\left\|\chi \chi_{\delta} v ; \mathscr{H}\right\| \leqslant c_{\delta}\|v ; \mathscr{H}\|$. Поскольку пространство $\mathscr{H}$ получено пополнением линеала $C_{c}^{\infty}(\bar{\Omega} \backslash \mathscr{O})^{k}$, дальнейшее доказательство можно проводить для гладкой вектор-функции $v$ с носителем, расположенным на множестве $\overline{\Xi_{\delta}}$ и отделенным от точки $\mathscr{O}$.

Пусть $p^{1}, \ldots, p^{d}$ - базис в линеале (6), причем $p^{j}(y)=\left(p_{1}^{j}(y), \ldots, p_{k}^{j}(y)\right)^{\top}$, $p_{h}^{j}$ - однородный полином степени $q_{j}, q_{1} \leqslant \cdots \leqslant q_{d}, \mathbf{a} \mathbf{p}=\left(p^{1}, \ldots, p^{d}\right)$ - матрица размера $k \times d$. Вектор-функцию $v$ представим в виде

$$
v(y, z)=v^{\perp}(y, z)+\sum_{j=1}^{d} V_{j}(z) p^{j}(y),
$$

где

$$
\begin{aligned}
V(z) & =\left(V_{1}(z), \ldots, V_{d}(z)\right)^{\top} \\
& =\mathbf{P}(z)^{-1} \int_{\omega(z)} \mathbf{p}(y)^{\top} \mathscr{D}\left(e_{n}\right)^{\top} \mathscr{D}\left(e_{n}\right) v(y, z) d y \quad \text { при п. в. } z \in(0, d), \\
\mathbf{P}(z) & =\int_{\omega(z)} \mathbf{p}(y)^{\top} \mathscr{D}\left(e_{n}\right)^{\top} \mathscr{D}\left(e_{n}\right) \mathbf{p}(y) d y=z^{(1+\gamma)(n-1)} \mathbf{Q}(z)^{\top} \mathbf{P}(1) \mathbf{Q}(z), \\
\mathbf{Q}(z) & =\operatorname{diag}\left\{z^{(1+\gamma) q_{1}}, \ldots, z^{(1+\gamma) q_{d}}\right\} .
\end{aligned}
$$

Поскольку ранг матрицы $\mathscr{D}\left(e_{n}\right)$ равен $k$, полиномиальные столбцы $\mathscr{D}\left(e_{n}\right) p^{1}, \ldots$, $\mathscr{D}\left(e_{n}\right) p^{d}$ высоты $N$ линейно независимы в пространстве $L_{2}(\omega)^{k}$, т. е. $\mathbf{P}(1)$ есть $(d \times d)$-матрица Грама, эрмитова и положительно определенная.

Согласно формулам (19), (20) и (6),

$$
\mathscr{D}\left(\nabla_{x}\right) v(x)=\mathscr{D}\left(\nabla_{y}, 0\right) v^{\perp}(x)+\mathscr{D}\left(e_{n}\right) \partial_{z} v^{\perp}(x)+\mathscr{D}\left(e_{n}\right) \mathbf{p}(y) \partial_{z} V(z) .
$$


Кроме того, выполнено условие ортогональности

$$
\int_{\omega(z)} \mathbf{p}(y)^{\top} \mathscr{D}\left(e_{n}\right)^{\top} \mathscr{D}\left(e_{n}\right) v^{\perp}(y, z) d y=0 \in \mathbb{C}^{d} \quad \text { при п.в. } z \in(0, \delta) .
$$

Соотношения (21) и (16) показывают, что

$$
\begin{aligned}
& c \mathscr{D} \int_{\Xi_{\delta}}\left|\mathscr{D}\left(\nabla_{x}\right) v(x)\right|^{2} d x \geqslant \int_{\Xi_{\delta}}\left|\mathscr{D}\left(\nabla_{y}, 0\right) v(x)\right|^{2} d x+\int_{\Xi_{\delta}}\left|\mathscr{D}\left(e_{n}\right) \partial_{z} v(x)\right|^{2} d x \\
& \geqslant \frac{1}{2} \int_{\Xi_{\delta}}\left|\mathscr{D}\left(\nabla_{x}\right) v^{\perp}(x)\right|^{2} d x+\int_{\Xi_{\delta}}\left|\mathscr{D}\left(e_{n}\right) \mathbf{p}(y) \partial_{z} V(z)\right|^{2} d x \\
&+2 \int_{\Xi_{\delta}} \partial_{z} v^{\perp}(x) \mathscr{D}\left(e_{n}\right)^{\top} \mathscr{D}\left(e_{n}\right) \mathbf{p}(y) \partial_{z} V(z) d x=: \frac{1}{2} I_{1}+I_{2}+2 I_{3} .
\end{aligned}
$$

Далее в лемме 5 будут проверены неравенства

$$
\begin{aligned}
& \left\|\nabla_{x} v^{\perp} ; L_{2}\left(\Xi_{\delta}\right)\right\|^{2}+\left\|z^{-1-\gamma} v^{\perp} ; L_{2}\left(\Xi_{\delta}\right)\right\|^{2}+\left\|z^{-(1+\gamma) / 2} v^{\perp} ; L_{2}\left(\partial \Xi_{\delta} \cap \partial \Omega\right)\right\|^{2} \\
& \quad \leqslant c\left\|\mathscr{D}\left(\nabla_{x}\right) v^{\perp} ; L_{2}\left(\Xi_{\delta}\right)\right\|^{2} \\
& \int_{0}^{\delta} z^{(1+\gamma)\left(n-1+2 q_{j}\right)}\left(\left|\partial_{z} V_{j}(z)\right|^{2}+z^{-2}\left|V_{j}(z)\right|^{2}\right) d z \\
& \quad \leqslant c\left\|\mathscr{D}\left(e_{n}\right) \mathbf{p}(y) \partial_{z} V ; L_{2}\left(\Xi_{\delta}\right)\right\|^{2}, \quad j=1, \ldots, d
\end{aligned}
$$

которые предоставляют оценки снизу интегралов $I_{1}$ и $I_{2}$. Рассмотрим интеграл $I_{3}$.

При помощи растяжения координат $y \mapsto \eta($ см. формулу $(1))$ и условия ортогональности (22) получаем равенство

$$
\begin{aligned}
0 & =\frac{\partial}{\partial z} \int_{\omega(z)} v^{\perp}(y, z)^{\top} \mathscr{D}\left(e_{n}\right)^{\top} \mathscr{D}\left(e_{n}\right) \mathbf{p}(y) d y \\
& =\frac{\partial}{\partial z}\left(\gamma^{n-1} z^{\gamma(n-1)} \int_{\omega} v^{\perp}\left(z^{1+\gamma} \eta, z\right)^{\top} \mathscr{D}\left(e_{n}\right)^{\top} \mathscr{D}\left(e_{n}\right) \mathbf{p}\left(z^{1+\gamma} \eta\right) d \eta\right) \quad \text { при п.в. } z \in(0, d) .
\end{aligned}
$$

Производя дифференцирование подынтегрального выражения и обращаясь к определениям (20), заключаем, что

$$
\begin{aligned}
& \left|\int_{\omega(z)} \partial_{z} v^{\perp}(y, z)^{\top} \mathscr{D}\left(e_{n}\right)^{\top} \mathscr{D}\left(e_{n}\right) \mathbf{p}(y) d y \partial_{z} V(z)\right| \\
& \leqslant c \sum_{j=1}^{J} \int_{\omega(z)}\left(z^{\gamma}\left|\nabla_{y} v^{\perp}(y, z)\right|+z^{-1}\left|v^{\perp}(y, z)\right|\right) d y z^{q_{j}(1+\gamma)}\left|\partial_{z} V_{j}(z)\right| \quad \text { при п.в. } z \in(0, d) .
\end{aligned}
$$

Таким образом, перенося вес $z^{\gamma}$ от первого сомножителя ко второму, получаем

$$
\begin{aligned}
\left|I_{3}\right| \leqslant c\left(\int_{\partial \Xi_{\delta}}\left(\left|\nabla_{y} v^{\perp}(y, z)\right|^{2}+z^{-2(1+\gamma)}\left|v^{\perp}(y, z)\right|^{2}\right) d s_{x}\right)^{1 / 2} \\
\quad \times\left(\sum_{j=1}^{d} \int_{0}^{\delta} z^{2 \gamma} z^{(1+\gamma)\left(n-1+2 q_{j}\right)}\left|\partial_{z} V_{j}(z)\right|^{2} d z\right)^{1 / 2} \leqslant c \delta^{\gamma}\left(I_{1} I_{2}\right)^{1 / 2} .
\end{aligned}
$$


Выбрав число $\delta>0$ достаточно малым, выводим из соотношений (23)-(26) неравенство

$$
\begin{gathered}
\left\|z^{-1-\gamma} v^{\perp} ; L_{2}\left(\Xi_{\delta}\right)\right\|^{2}+\sum_{j=1}^{d}\left\|z^{-1+(1+\gamma)\left(n-1+2 q_{j}\right) / 2} V_{j} ; L_{2}(0, \delta)\right\|^{2} \\
\leqslant c\left(I_{1}+I_{2}\right) \leqslant c\left\|\mathscr{D}\left(\nabla_{x}\right) v ; L_{2}\left(\Xi_{\delta}\right)\right\|^{2},
\end{gathered}
$$

которое при помощи представления (19) и формул (20) легко преобразуется в искомую оценку (17), так как возникшая норма функции $z^{-1+(1+\gamma)\left(n-1+2 q_{j}\right) / 2} V_{j}$ в пространстве $L_{2}(0, \delta)$ эквивалентна норме $\left\|z^{-1+(1+\gamma) q_{j}} V_{j} ; L_{2}\left(\Xi_{\delta}\right)\right\|$ и больше величины $c\left\|z^{-1} p^{j} V_{j} ; L_{2}\left(\Xi_{\delta}\right)\right\|$, а $z$ и $r$ - эквивалентные бесконечно малые на множестве $\Xi_{\delta}$.

Оператор вложения $\mathscr{H} \subset L_{2}(\Omega)^{k}$ представим в виде суммы компактного оператора (на множестве $\Omega \backslash \Xi_{\delta}$; см. неравенство (18)) и оператора с нормой, не превосходящей величины $c \delta$ (на кончике $\Xi_{\delta}$ пика; см. неравенство (17) с учетом соотношений $r \leqslant\left(z^{2}+c z^{2(1+\gamma)}\right)^{1 / 2}, z<\delta$ на $\left.\Xi_{\delta}\right)$. Поскольку число $\delta>0$ произвольно, упомянутое вложение компактно, т.е. условие в п. 1) теоремы 1 является достаточным. Необходимость условия обеспечена уже доказанным вторым утверждением теоремы. Осталось убедиться в справедливости следующей леммы.

Лемма 5. 1) Для функций $V_{1}, \ldots, V_{d} \in C_{c}^{\infty}(0, \delta)$ справедливо неравенство (25).

2) Если вектор-функиия $v^{\perp} \in C^{\infty}\left(\overline{\Xi_{\delta}}\right)^{k}$ обращается в нуль при $z=\delta$ и подчинена условиям ортогональности (22), то имеет место неравенство (24).

Доказательство. В силу формул (20) и (23) имеем

$$
\begin{aligned}
I_{2} & =\int_{0}^{\delta} z^{(1+\gamma)(n-1)} \sum_{j, m=1}^{d} \int_{\omega} p^{j}(\eta)^{\top} \mathscr{D}\left(e_{n}\right)^{\top} \mathscr{D}\left(e_{n}\right) p^{m}(\eta) d \eta \partial_{z} V_{j}(z) \partial_{z} V_{m}(z) d z \\
& =\int_{0}^{\delta} z^{(1+\gamma)(n-1)}\left(\mathbf{Q}(z) \partial_{z} V(z)\right)^{\top} \mathbf{P}(z) \mathbf{Q}(z) \partial_{z} V(z) d z \\
& \geqslant c_{\mathbf{P}} \sum_{j=1}^{d} \int_{0}^{\delta} z^{\beta_{j}+1}\left|\partial_{z} V_{j}(z)\right|^{2} d z \geqslant c_{\mathbf{P}} \sum_{j=1}^{d} \beta_{j}^{-2} \int_{0}^{\delta} z^{\beta_{j}-1}\left|V_{j}(z)\right|^{2} d z .
\end{aligned}
$$

Здесь применено неравенство Харди

$$
\int_{0}^{\delta} z^{\beta-1}|V(z)|^{2} d z \leqslant 4 \beta^{-1} \int_{0}^{\delta} z^{\beta+1}\left|\partial_{z} V(z)\right|^{2} d z, \quad V \in C^{1}[0, \delta], V(\delta)=0,
$$

с показателями $\beta_{j}=(1+\gamma)\left(n-1+2 q_{j}\right)-1>0, j=1, \ldots, d$. Соотношение $(25)$ установлено.

Проверим теперь соотношение (24). Замена координат

$$
x=(y, z) \mapsto(\eta, \zeta)=\left(z^{-1-\gamma} y,-\gamma^{-1} z^{-\gamma}\right)
$$

преобразует множество $\Xi_{\delta}$ в полуцилиндр

$$
Q=\left\{(\eta, \zeta): \eta \in \omega, \zeta<-T:=-\gamma^{-1} \delta^{-\gamma}\right\}
$$

Кроме того,

$$
\nabla_{y}=z^{-1-\gamma} \nabla_{\eta}, \quad \partial_{z}=z^{-1-\gamma}\left(\partial_{\zeta}-(1+\gamma) z^{\gamma} \eta^{\top} \nabla_{\eta}\right),
$$


а значит, для вектор-функции $(\eta, \zeta) \mapsto \mathbf{v}^{\perp}(\eta, \zeta)=v^{\perp}(y, z)$ выполнено равенство

$$
I_{1}=\int_{Q} z^{(1+\gamma)(n-2)}\left|\mathscr{D}\left(\nabla_{\eta}, \partial_{\zeta}\right) \mathbf{v}^{\perp}(\eta, \zeta)-(1+\gamma) z^{\gamma} \mathscr{D}\left(e_{n} \eta^{\top} \nabla_{\eta}\right) \mathbf{v}^{\perp}(\eta, \zeta)\right|^{2} d \eta d \zeta
$$

Рассечем полуцилиндр $Q$ на равные части $Q^{m}=\{(\eta, \zeta) \in Q: \zeta+T \in$ $(-m, 1-m)\}, m=1,2, \ldots$. Благодаря условиям ортогональности (22) справедливо соотношение

$$
\int_{Q_{m}} \mathbf{p}(\eta)^{\top} \mathscr{D}\left(e_{n}\right)^{\top} \mathscr{D}\left(e_{n}\right) \mathbf{v}^{\perp}(\eta, \zeta) d \eta d \zeta=0 \in \mathbb{C}^{d}
$$

Согласно [5, гл. 5], выполнено обобщенное неравенство Корна

$$
\left\|\mathbf{v}^{\perp} ; H^{1}\left(Q^{m}\right)\right\|^{2} \leqslant c\left(\left\|\mathscr{D}\left(\nabla_{\eta}, \partial_{z}\right) \mathbf{v}^{\perp} ; L_{2}\left(Q^{m}\right)\right\|^{2}+\left\|\mathbf{v}^{\perp} ; L_{2}\left(Q^{m}\right)\right\|^{2}\right),
$$

которое при помощи теоремы об эквивалентных нормировках можно преобразовать в неравенство

$$
\left\|\mathbf{v}^{\perp} ; H^{1}\left(Q^{m}\right)\right\|^{2} \leqslant c\left(\left\|\mathscr{D}\left(\nabla_{\eta}, \partial_{z}\right) \mathbf{v}^{\perp} ; L_{2}\left(Q^{m}\right)\right\|^{2}+\left|\mathbf{F}\left(\mathbf{v}^{\perp}\right)\right|^{2}\right),
$$

где $\mathbf{F}: L_{2}(\Omega)^{k} \rightarrow \mathbb{C}^{d}-$ любой непрерывный линейный функционал, подчиненный следующему требованию:

$$
\mathbf{F}(p)=0 \quad \text { при некотором } p \in \mathscr{P} \Longrightarrow p=0 \text {. }
$$

Постоянные $c$ в формулах (30) и (31) не зависят ни от вектор-функции $\mathbf{v}^{\perp}$, ни от номера $m$ цилиндра $Q^{m}$ единичной высоты.

Приведем непосредственную проверку эквивалентности норм (30) и (31). Достаточно убедиться в справедливости неравенства

$$
\left\|\mathbf{v}^{\perp} ; L_{2}\left(Q^{m}\right)\right\|^{2} \leqslant c\left(\left\|\mathscr{D}\left(\nabla_{\eta}, \partial_{z}\right) \mathbf{v}^{\perp} ; L_{2}\left(Q^{m}\right)\right\|^{2}+\left|\mathbf{F}\left(\mathbf{v}^{\perp}\right)\right|^{2}\right) .
$$

Предположим, что не существует общей постоянной $c$. Тогда найдется такая последовательность $\left\{\mathbf{v}^{q}\right\}$ элементов пространства $H^{1}\left(Q^{m}\right)^{k}$, что $\left\|\mathbf{v}^{q} ; L_{2}\left(Q^{m}\right)\right\|^{2}=1, \quad\left\|\mathscr{D}\left(\nabla_{\eta}, \partial_{z}\right) \mathbf{v}^{q} ; L_{2}\left(Q^{m}\right)\right\| \rightarrow 0, \quad\left|\mathbf{F}\left(\mathbf{v}^{q}\right)\right| \rightarrow 0 \quad$ при $q \rightarrow \infty$. Ввиду оценки (30) нормы $\left\|\mathbf{v}^{q} ; H^{1}\left(Q^{m}\right)\right\|$ равномерно ограничены, т. е. некоторая подпоследовательность имеет предел $\mathbf{v}^{\infty}$, слабый в $H^{1}\left(Q^{m}\right)^{k}$ и сильный в $L_{2}\left(Q^{m}\right)^{k}$. При этом $\mathscr{D}\left(\nabla_{\eta}, \partial_{\zeta}\right) \mathbf{v}^{\infty}=0$ и $\mathbf{F}\left(\mathbf{v}^{\infty}\right)=0$, а значит, формула (6) показывает, что $\mathbf{v}^{\infty} \in \mathscr{P}$, т. е. $\mathbf{v}^{\infty}=0$ в силу свойства (32). Последнее равенство невозможно из-за соотношения $\left\|\mathbf{v}^{\infty} ; L_{2}\left(Q^{m}\right)\right\|=1$, обусловленного сильной сходимостью последовательности $\left\{\mathbf{v}^{q}\right\}$ по норме пространства $L_{2}(\Omega)^{k}$. Полученное противоречие устанавливает справедливость неравенства (31).

Возьмем в качестве $\mathbf{F}\left(\mathbf{v}^{\perp}\right)$ левую часть формулы $(29)$; понятно, что требование (32) соблюдено в силу определений (20). Неравенство (31) вместе со следовым неравенством [7] гарантируют, что

$$
\begin{gathered}
\left\|\nabla_{(\eta, \zeta)} \mathbf{v}^{\perp} ; L_{2}\left(Q^{m}\right)\right\|^{2}+\left\|\mathbf{v}^{\perp} ; L_{2}\left(Q^{m}\right)\right\|^{2}+\left\|\mathbf{v}^{\perp} ; L_{2}\left(\partial Q^{m} \cap \partial Q\right)\right\|^{2} \\
\leqslant c\left\|\mathscr{D}\left(\nabla_{\eta}, \partial_{z}\right) \mathbf{v}^{\perp} ; L_{2}\left(Q^{m}\right)\right\|^{2} .
\end{gathered}
$$

Постоянная $c$ не зависит ни от $m$, ни от $\mathbf{v}^{\perp}$. Умножим обе части неравенства (33) на $z_{m}^{(1+\gamma)(n-2)}$, где $\gamma z_{m}^{-\gamma}=T+m$. Поскольку $z_{m}<z<(1+c) z_{m}$ при $(\eta, \zeta) \in$ $Q_{m}$, множители можно занести под знаки норм, превратив их (и множители, и 
нормы) в весовые. В результате суммирование по $m=1,2, \ldots$ преобразованных неравенств (33) приводит к соотношению

$$
\begin{aligned}
& \left\|z^{(1+\gamma)(n-2) / 2} \nabla_{(\eta, \zeta)} \mathbf{v}^{\perp} ; L_{2}(Q)\right\|^{2}+\left\|z^{(1+\gamma)(n-2) / 2} \mathbf{v}^{\perp} ; L_{2}(Q)\right\|^{2} \\
& \quad+\left\|z^{(1+\gamma)(n-2) / 2} \mathbf{v}^{\perp} ; L_{2}(\partial Q)\right\|^{2} \leqslant c\left\|z^{(1+\gamma)(n-2) / 2} \mathscr{D}\left(\nabla_{\eta}, \partial_{z}\right) \mathbf{v}^{\perp} ; L_{2}(Q)\right\|^{2} .
\end{aligned}
$$

Коэффициент $z^{\gamma}$ при втором слагаемом из подынтегрального выражения в (28) может быть сделан малым за счет выбора $\delta$, а значит, правая часть соотношения (34) мажорируется величиной $c I_{1}$. Переход к координатам $(y, z)$ согласно формулам (27) превращает модифицированную указанным способом оценку (34) в искомое неравенство (24).

\section{§5. Обсуждение, примеры и гипотезы}

Явная формула (1) для заострения введена лишь для упрощения изложения - определение можно сделать, например, таким:

$$
z>0, \quad H(z)^{-1} y \in \omega .
$$

Здесь $H$ - положительная функция, удовлетворяющая соотношениям

$$
\left|z^{-1-\gamma} H(z)-H_{0}\right|+\left|z^{-\gamma} \partial_{z} H(z)-(1+\gamma) H_{0}\right| \leqslant c z^{\delta}, \quad H_{0}>0, \delta>0,
$$

а координаты $\eta$ и $\zeta$ из замены (27) выглядят следующим образом:

$$
\eta=H(z)^{-1} y \in \omega, \quad \zeta=-\int_{z}^{R} H(\mathfrak{z})^{-1} d \mathfrak{z} .
$$

Если $\gamma \geqslant 1$ и, значит, пик достаточно острый, теорема 1 предоставляет критерий образования непустого существенного (непрерывного; см. замечание 2) спектра задачи (4). При $\gamma \in(0,1)$ в теореме содержится только признак, так как она не дает информации о спектре при $\gamma \in\left(0, J^{-1}\right)$. Автор выдвигает гипотезу: при $\gamma \in\left(0, J^{-1}\right)$, где $J$ - максимальная степень полинома вида $(13)$ в линеале (6), спектр оказывается дискретным. Еще одна гипотеза связана со строением непрерывного спектра в предельном случае $\gamma=J^{-1}$, а именно: существует такой «порог» $\lambda_{\bullet}>0$, что на полуинтервале $\left[0, \lambda_{\bullet}\right)$ расположен дискретный спектр, а луч $\left[\lambda_{\bullet}, \infty\right)$ представляет собой непрерывный спектр. Наконец, автор подозревает, что в случае $\gamma>J^{-1}$ вся замкнутая вещественная полуось покрыта непрерывным спектром задачи (4).

Пример 6. Пусть $k=n=3, B(x)=\rho(x) \mathbb{I}_{3}, \mathbb{I}_{k}$ - единичная матрица размера $k \times k$ и

$$
\mathscr{D}\left(\nabla_{x}\right)^{\top}=\left(\begin{array}{cccccc}
\partial_{1} & 0 & 2^{-1 / 2} \partial_{2} & 2^{-1 / 2} \partial_{z} & 0 & 0 \\
0 & \partial_{2} & 2^{-1 / 2} \partial_{1} & 0 & 2^{-1 / 2} \partial_{z} & 0 \\
0 & 0 & 0 & 2^{-1 / 2} \partial_{1} & 2^{-1 / 2} \partial_{2} & \partial_{z}
\end{array}\right),
$$

где $\partial_{i}=\partial / \partial y_{i}, i=1,2$. В этом случае соотношения $(2),(3)$ образуют задачу линейной теории упругости, записанную в матричной (а не тензорной) форме (см., например, [8]), причем $u$ - столбец смещений, $\mathscr{D}\left(\nabla_{x}\right) u$ и $A \mathscr{D}\left(\nabla_{x}\right) u$ - столбцы деформаций и напряжений, $A$ - вещественная матрица упругих модулей, симметрическая и положительно определенная, и $\rho>0$ - плотность материала. Линеал (6) составлен из жестких смещений $a+b \times x$, где $a$ и $b$ - постоянные столбцы в $\mathbb{R}^{3}$ и символ $\times$ обозначает векторное произведение. В $\mathscr{P}$ существуют нетривиальные линейные вектор-функции всех трех аргументов, и поэтому 
$J=1$, а задача (2), (3) о свободных колебаниях упругого тела с острым пиком (1) имеет непрерывный спектр. Эта задача допускает более детальное исследование [9 $]^{1)}$, опирающееся на весовое неравенство Корна [10] и асимптотические анзацы в теории тонких стержней (см., например, [8, гл. 5]) и частично подтверждающее для конкретного примера сформулированные гипотезы. Именно, при $\gamma \in(0,1)$ задача обладает дискретным спектром (12) и в случае $\gamma=1$ луч $\left[\lambda_{\ddagger}, \infty\right)$ лежит на непрерывном спектре; здесь $\lambda_{\ddagger}-$ некоторое число, зависящее от сечения $\omega$, матрицы $A(\mathscr{O})$ и скаляра $\rho(\mathscr{O})$. Соответствующие вычисления и окончательные формулы очень громоздки - ограничимся упрощенным случаем изотропного однородного материала, для которого $\rho>0-$ постоянная,

$$
A=\left(\begin{array}{cccccc}
\boldsymbol{\lambda}+2 \boldsymbol{\mu} & \boldsymbol{\lambda} & 0 & 0 & 0 & \boldsymbol{\lambda} \\
\boldsymbol{\lambda} & \boldsymbol{\lambda}+2 \boldsymbol{\mu} & 0 & 0 & 0 & \boldsymbol{\lambda} \\
0 & 0 & 2 \boldsymbol{\mu} & 0 & 0 & 0 \\
0 & 0 & 0 & 2 \boldsymbol{\mu} & 0 & 0 \\
0 & 0 & 0 & 0 & 2 \boldsymbol{\mu} & 0 \\
\boldsymbol{\lambda} & \boldsymbol{\lambda} & 0 & 0 & 0 & \boldsymbol{\lambda}+2 \boldsymbol{\mu}
\end{array}\right),
$$

а $\boldsymbol{\lambda} \geqslant 0$ и $\boldsymbol{\mu}>0$ - постоянные Ламе. Если центр тяжести плоской фигуры $\boldsymbol{\omega}$ совпадает с началом декартовой системы координат $\eta$, а оси $\eta_{1}$ и $\eta_{2}$ направлены вдоль главных осей тензора инерции фигуры, то

$$
\lambda_{\ddagger}=\frac{49 \cdot 25}{\rho \operatorname{mes}_{2} \omega} \boldsymbol{\mu} \frac{3 \boldsymbol{\lambda}+2 \boldsymbol{\mu}}{\boldsymbol{\lambda}+\boldsymbol{\mu}} \mathbf{I}(\omega), \quad \mathbf{I}(\omega)=\min \left\{\int_{\omega} \eta_{1}^{2} d \eta, \int_{\omega} \eta_{2}^{2} d \eta\right\} .
$$

Отметим также, что для изотропного однородного тела $\Omega$ с осью вращения $z$ на непрерывном спектре, включающем луч $\left[\lambda_{\ddagger}, \infty\right)$, расположен бесконечный набор элементов точечного спектра, т. е. собственных чисел.

Теорема 1 соотносит свойства спектра с полуторалинейной формой $a(\cdot, \cdot ; \Omega)$, но никак не с самим дифференциальным оператором $\mathscr{L}\left(x, \nabla_{x}\right)$. Так, билинейной форме

$$
\widetilde{a}(u, v ; \Omega)=\boldsymbol{\mu} \sum_{j=1}^{3}\left(\nabla_{x} u_{j}, \nabla_{x} v_{j}\right)_{\Omega}+(\boldsymbol{\lambda}+\boldsymbol{\mu})\left(\nabla_{x}^{\top} u, \nabla_{x}^{\top} v\right)_{\Omega}
$$

отвечает тот же матричный дифференциальный оператор $\mathscr{L}\left(\nabla_{x}\right)$, что и форме $a(u, v ; \Omega)$, построенной по матрицам (35) и (36) согласно определению (4). Линеал $\widetilde{\mathscr{P}}$, порожденный формой $(37)$, состоит лишь из постоянных векторов, т. е. при любом $\gamma>0$ по п. 1) теоремы 1 дискретным оказывается спектр задачи с оператором $\mathscr{L}\left(\nabla_{x}\right)$, а также с (нефизическими) функционалом энергии $(37)$ и условиями Неймана, проистекающими из формулы Грина. По п. 2) теоремы 1 у настоящей задачи теории упругости (пример 6) спектр не является дискретным при $\gamma \geqslant 1$.

В случае анизотропного строения линеала $\mathscr{P}$ появление существенного спектра зависит от направления пика. Например, при замене матрицы (35) матрицей

$$
\mathscr{D}\left(\nabla_{x}\right)^{\top}=\left(\begin{array}{cccccccc}
\partial_{1} & 0 & 2^{-1 / 2} \partial_{2} & 0 & 0 & 0 & 0 & \partial_{z} \\
0 & \partial_{2} & 2^{-1 / 2} \partial_{1} & 0 & 0 & 0 & \partial_{z} & 0 \\
0 & 0 & 0 & \partial_{1} & \partial_{2} & \partial_{z} & 0 & 0
\end{array}\right)
$$

1) Для плоского изотропного тела с показателем заострения $\gamma=1$ доказательство появления непрерывного спектра анонсировано И. В. Камоцким. 
и расширении положительно определенной симметрической матрицы (36) до размера $8 \times 8$ линеал (6) содержит линейные вектор-функции переменных $y_{1}$ и $y_{2}$, но не переменной z. Поэтому в случае острого пика (1) спектр оказывается дискретным, однако непустой существенный спектр возникает при ненулевом угле между осью пика и осью аппликат (изменяем область, сохраняя декартову систему координат и оператор $\mathscr{L})$.

Пример 7. Пусть $n=2$ и матрица $\mathscr{D}\left(\nabla_{x}\right)$ составлена из $k+1$ строк длины $k$ :

$$
\left(\begin{array}{cccccc}
\partial_{y} & 0 & 0 & \ldots & 0 & 0 \\
-\partial_{z} & \partial_{y} & 0 & \ldots & 0 & 0 \\
0 & -\partial_{z} & \partial_{y} & \ldots & 0 & 0 \\
\vdots & \vdots & \vdots & \ddots & \vdots & \vdots \\
0 & 0 & 0 & \ldots & \partial_{y} & 0 \\
0 & 0 & 0 & \ldots & -\partial_{z} & \partial_{y} \\
0 & 0 & 0 & \ldots & 0 & -\partial_{z}
\end{array}\right)
$$

Нетрудно убедиться в том, что полином

$$
P(y, z)=\left(\frac{z^{k-1}}{(k-1) ! 0 !}, \frac{z^{k-2} y}{(k-2) ! 1 !}, \frac{z^{k-3} y^{2}}{(k-3) ! 2 !}, \ldots, \frac{y^{k-1}}{0 !(k-1) !}\right)^{\top}
$$

имеющий степень $J=k-1$, аннулируется дифференциальным оператором (38). Таким образом, при любом $\gamma>0$ можно построить билинейную форму и матричный дифференциальный оператор, для которых спектр задачи (2), (3) в области с пиком (1) не является дискретным.

Пример 8. Пьезоэлектрическое тело $\Omega$ описывается (см., например, книгу [11]) при помощи задачи (2), (3), в которой $k=4$ и $N=9$, причем $u^{(1)}=$ $\left(u_{1}, u_{2}, u_{3}\right)^{\top}$ - столбец смещений, $u^{(2)}=u_{4}$ - электрический потенциал, а операторы $\mathscr{L}$ и $\mathscr{N}$ сооружены из следующих матриц, имеющих размеры $4 \times 9$ и $9 \times 9$ :

$$
\mathscr{D}\left(\nabla_{x}\right)^{\top}=\left(\begin{array}{cc}
\mathscr{D}^{(11)}\left(\nabla_{x}\right)^{\top} & \mathbb{I}_{3} \\
0,0,0,0,0,0 & \nabla_{x}^{\top}
\end{array}\right), \quad A=\left(\begin{array}{cc}
A^{(11)} & A^{(12)} \\
-A^{(21)} & A^{(22)}
\end{array}\right) .
$$

Здесь $A^{(11)}$ и $A^{(22)}$ - симметрические и положительно определенные матрицы размера $6 \times 6$ и $3 \times 3$ соответственно, $A^{(12)}=\left(A^{(21)}\right)^{\top}$, а $\mathscr{D}^{(11)}\left(\nabla_{x}\right)^{\top}$ - оператор (35). В соответствии с физической интерпретацией задачи матрица $A$ не является симметрической, а матрица $B=\operatorname{diag}\{\rho, \rho, \rho, 0\}-$ положительной. Тем не менее приемы введения комплексного электрического потенциала $\varphi=i u_{4}[4$, пример 1.13] и отщепления скалярной задачи для $\varphi$ [12], в которой отсутствует спектральный параметр, приводят задачу пьезоэлектричества к виду, позволяющему перенести на нее результаты о спектре задачи теории упругости из примера 6. Интересно, что общие свойства спектра безразличны к тому, какие краевые условия, (3) или

$$
e_{j}^{\top} \mathscr{N}\left(x, \nabla_{x}\right) u(x)=0, \quad j=1,2,3, \quad u_{4}(x)=0, \quad x \in \partial \Omega \backslash \mathscr{O},
$$

заданы на границе тела (соотношения (39) означают, что поверхность тела свободна от внешних механических нагрузок и электрически заземлена, а условия (3) соответствуют тому, что тело помещено в вакуум). 
Замечание 9. Пусть столбцы $a^{1}, \ldots, a^{k}$ образуют базис в пространстве $\mathbb{C}^{k}$. Если оказалось, что при некотором $m \in\{0, \ldots, k\}$ любой столбец полиномов $p=\left(p_{1}, \ldots, p_{k}\right)^{\top} \in \mathscr{P}$, для которого $a_{1}^{j} p_{1}(y, z)+\cdots+a_{k}^{j} p_{k}(y, z)-$ нулевой вектор при всех $j=m+1, \ldots, k$ и $(y, z) \in \mathbb{R}^{n}$, не зависит от переменной $z$, то теорема 1 сохраняет силу при замене условий Неймана (3) смешанными краевыми условиями

$$
\begin{aligned}
\left(a^{q}\right)^{\top} \mathscr{N}\left(x, \nabla_{x}\right) u(x) & =0, & & q=1, \ldots, m, \\
\left(a^{j}\right)^{\top} u(x) & =0, & j & =m+1, \ldots, k, x \in \partial \Omega \backslash \mathscr{O} .
\end{aligned}
$$

Дело в том, что последние условия в (40) являются устойчивыми, и задача (4) ставится на подпространстве $\mathscr{H}^{0}=\left\{u \in \mathscr{H}:\left(a^{j}\right)^{\top} u(x)=0, x \in \partial \Omega \backslash \mathscr{O}, j=\right.$ $m+1, \ldots, k\}$, которое выделяет линеал $\mathscr{P}^{0}=\mathscr{P} \cap \mathscr{H}^{0}$, свободный по предположению от векторных полиномов, зависящих от переменной $z$. Все предшествующие доказательства приспосабливаются к задаче (2), (40). Заметим, что при $m=0$ линеал $\mathscr{P}^{0}$ тривиален, а условия (40) означают равенство $u(x)=0$, $x \in \partial \Omega \backslash \mathscr{O}$. Впрочем, спектр получившейся задачи Дирихле дискретен по простым причинам.

\section{ЛитерАТУРА}

[1] В. Г. Мазья, Пространства Соболева, изд-во Ленинград. ун-та, Л., 1985.

[2] М. Ш. Бирман, М. З. Соломяк, Спектральная теория самосопряженных операторов в гильбертовом пространстве, изд-во Ленинград. ун-та, Л., 1980.

[3] С. А. Назаров, Самосопряжсенные эллиптические краевые задачи. Полиномиальное свойство и формально положительные операторы, в кн.: Проблемы матем. анализа. Вып. 16, изд-во СПбГУ, СПб, 1997, 167-192.

[4] С. А. Назаров, Полиномиальное свойство самосопрлженных эллиптических краевых задач и алгебрачческое описание их атрибутов, УМН, 54:5 (1999), 77-142.

[5] J. Nečas, Les méthodes directes en théorie des équations elliptiques, Masson-Academia, Paris-Prague, 1967.

[6] V. A. Kozlov, V. G. Maz'ya, J. Rossmann, Elliptic Boundary Value Problems in Domains with Point Singularities, Amer. Math. Soc., Providence, 1997.

[7] О. А. Ладыженская, Краевые задачи математической физики, Наука, М., 1973.

[8] С. А. Назаров, Асимптотическая теория тонких пластин и стержней. Понижение размерности и интегральные оценки, Научная книга, Новосибирск, 2001.

[9] С. А. Назаров, О спектре задачи теории упругости для тела пикообразной формы, Сиб. матем. ж., 49:5 (2008), 1105-1127.

[10] С. А. Назаров, Весовые неравенства Корна на параболоидальных областях, Матем. заметки, 62:5 (1997), 751-765; исправление: Матем. заметки, 63:4 (1998), 640.

[11] В. З. Партон, Б. А. Кудрявцев, Электроупругость пъезоэлектрических и электропроводных тел, Наука, М., 1988.

[12] С. А. Назаров, Равномерные оченки остатков в асимптотических разложениях решений задачи о собственных колебаниях пъезоэлектрической пластины, в кн.: Проблемы матем. анализа. Вып. 25, Научная книга, Новосибирск, 2003, 99-188.

ИПМаш РАН, Санкт-Петербург e-mail: serna@snark.ipme.ru,srgnazarov@yahoo.co.uk 